J. Management and Humanity Research

Vol. 4, 2020, 1-7

ISSN: 2582-7766 (online)

Published on 25 January 2021

www.researchmathsci.org

DOI: http://dx.doi.org/10.22457/jmhr.v04a04101

\title{
Business Models of Paying for Knowledge of Video Websites: A Case Study from China
}

\section{Dongxue Xie}

\author{
School of Electronic Information Engineering, Beijing Jiaotong University \\ Beijing 100044, China. \\ Email: $\underline{3338468665 @ q q . c o m}$
}

Received 2 November 2020; accepted 2 January 2021

\begin{abstract}
With the rapid development of the Internet, mobile payment has entered everyone's life. Users are using more and more frequently, and the usage scenarios are more and more extensive. The knowledge payment model of learning products on the website has emerged and developed. This paper is studying the problems encountered by its users in the process of paying for knowledge on video websites. And it analyzes and summarizes the current situation of paying for knowledge of video websites, and compares the promotion and restriction factors of the payment-knowledge business model of video websites. Taking the learning videos of three mainstream websites, namely Bilibili, iQiYi, and Tik Tok as examples, it compared the development status and existing problems of learning videos on the websites respectively, and finally put forward marketing suggestions for the development and improvement of video websites.
\end{abstract}

Keywords: Video website; knowledge learning; pay for knowledge

\section{Introduction}

At the beginning of the 21 st century, the world has undergone profound changes. The Internet has improved the way people learn. New vocabularies such as website learning, webcasting, and the Internet have emerged. The emergence of new technologies such as big data, cloud computing, Internet of Things, and 5G provides new ideas for users of video websites to learn. The changes of desktop interconnection, PC interconnection, mobile interconnection to the interconnection of everything confirm that one of the important features of the Internet is information. The rapid spread of the website provides rapid development for website learning, and also serves different learners to meet the needs of users.

In 2020, China Internet Network Information Center (CNNIC) released the 45th "Statistical Report on the Development of China's Internet". The report shows that as of March 2020, the number of netizens in my country is 904 million, the Internet penetration rate is $64.5 \%$. This fully proves the arrival of the mobile Internet era of the external environment. From this point of view, China's video website development market has opened up. The rapid development of Bilibili, Tik Tok and IQiYi and other video websites contains huge business opportunities. Some video websites have realized commercial realization through knowledge payment. However, there are few profitable 


\section{Dongxue Xie}

video websites, mainly due to the low differentiation of video content, the lack of innovation in the content itself, the rampant piracy of video and the imperfect payment system, etc., but the fundamental reason is that they do not understand the learning needs and motivations of users. The characteristics are different, and the services provided are also different. Therefore, it is particularly important to study the content quality of the website and the learning behavior of users for video websites.

\section{Learning videos from mainstream websites}

Learning video is mainly divided into four categories, namely "study in prestigious schools". This column contains public courses of famous universities. The second section is "study in colleges and universities". This section mainly contains courses from wellknown middle and high schools or tuition institutions in China. Educational courses are very popular. The third section is "Learning in Interest", which meets the interests and personalities of users, mainly focusing on popular science, humanities and science. The fourth section is "Learning in the Workplace", which mainly focuses on the selfimprovement of users.

The websites are mainly divided into three categories, Tik Tok, IQiYi and Bilibili. Tik Tok mainly focuses on short videos, which is a kind of fragmented learning; IQiYi is an entertainment video website with more film copyrights; B station Users mainly follow fan dramas and the second element. It is an interactive social learning website. In addition to the above three platforms, since 2019, many short video platforms have been working hard to create online platform courses, and have successively launched knowledge and education Support the plan, and explore the realization.

\subsection{Bilibili}

Bilibili, referred to as station B. Although Bilibili is mainly for users of the second dimension, nowadays, it is a treasured place for young people to learn. Many users first watched entertainment videos at station $\mathrm{B}$, and now they watch learning videos at station $\mathrm{B}$, as an important boost for self-study. This phenomenon represents the attitude of today's young users towards choosing to learn methods. Bilibili's official data shows that there are about 7,000 vertical interest circles in Station B, which started with twodimensional content and games and other entertainment culture. In 2019, 18.27 million people studied at Station B, which is equivalent to twice the number of college entrance examinations that year.

Users of contemporary video sites do not like the one-way output of duck-filling teaching. There are countless learning video content on the website, which makes the learning process easy and enjoyable while improving the learning effect. Station B is an interactive social learning platform. Take station B as an example. Although many people think station B is a treasure trove of learning, strictly to speak, station B will not have a "learning area" in the standard sense until 2020. Nearly 100 million young users at station B. They are not only users of station B watching dramas and watching game videos, but also pets, speeches, beauty, science, digital, handicraft and cars. Although the layers overlap, they do not interfere with each other. The knowledge learning field of station B involves language test courses, manual experience courses, literature courses, work experience courses, daily learning courses, etc, especially manual experience courses, language test courses and work experience courses. According to the needs of users of different websites, Bilibili's learning area is subdivided into four sections, namely "study 


\section{Business Models of Paying for Knowledge of Video Websites: A Case Study from China}

in prestigious schools". This section contains public courses of famous universities, such as "Accounting: Basic Introduction" by China Accounting Online School and "Organizational Rules for Survival in Crisis", etc. The second section is "study in colleges and universities". This column mainly includes courses from well-known domestic middle and high schools or tuition institutions. The most popular are education courses, such as "English" by Shanghai Middle School. The third section is "Learning in Interest", which meets the interests and personalities of users, mainly focusing on popular science, humanities and science. The fourth section is "Learning in the Workplace". Many workplace leaders will come to share their experiences and teach professional knowledge, as well as career planning courses and title examination courses for young people.

It can be found from the learning video that learning in a short period of time based on watching the video is not traditional one-way learning, but to create a social learning atmosphere through barrage and comment interaction. When users have questions during the learning process, they can directly ask questions on the barrage, and often get answers from other users or even UP owners. Sending the bullet screens and the interaction of the comment area makes learning that is boring, lonely, and difficult to persist without being supervised by you become lively and interesting, learn knowledge happily, and also encourage the UP master to persist in creating and sharing in station B. The desire for more users to see and learn knowledge.

In 2020, Station B has divided a special learning area "Keep learning at station B ". There is a free live broadcast of the course, which contains rich learning content and covers a lot of coverage. The age group is divided into four sections: middle school classroom, college classroom, high-quality courses and high school classroom. The Bilibili website has more users than educational institutions. It is not only students that watch learning videos or live broadcasts, but also office workers that improve themselves.

\subsection{Tik Tok}

Tik Tok Short Video is a social platform integrating music innovation and focusing on young people [1]. Users select songs online, shoot short videos, and publish the works after they are formed. According to their own preferences, other users can watch, like, comment, favorite, share, reward or long-press to choose not interested, etc. The video duration of Tik Tok can only be published for 15 seconds from the beginning of the longest that can be published up to 60 seconds, and grasp the duration of the user's attention. The software can also use the background big data analysis to understand the usage habits.

If someone asked you what you have in common with Tik Tok, Weishi and Kuaishou two years ago, you might be very confused, or the answer might be messy, but most people think they can't learn. But after 2018, "short video learning" is becoming the choice of more people. The Tik Tok learning short film means that the Tik Tok platform

disseminates the instructional video for less than 1 minute, focusing on the essence of the content, which is shorter, more refined and covers a wider range than ordinary microclasses. 


\section{Dongxue Xie}

Nowadays, learning short videos on Tik Tok is valuable resources for learning. These short videos refine complex knowledge into individual knowledge points, cater to the user's time fragmentation characteristics [1], and reduce the difficulty of psychological preparation for learners, You can learn with short videos anytime and anywhere, such as waiting for public transportation, subway, elevator, and other fragmented time. Some people use Tik Tok just to free themselves in their free time and watch funny videos to get happiness, but some users use their time of Tik Tok to look at Excel skills or test knowledge points and other learning knowledge. Since then, Tiktok has launched live classes of well-known domestic universities, such as the live class of Sun Yat-sen University. There is a section of knowledge category in Tik Tok's Creator Academy, where learning videos include education, workplace, mother and child, history, technology, emotion, etc.

When short videos satisfy users' content preferences, they also make them interested. Users will continue to watch them and better absorb knowledge. Tik Tok makes small knowledge points more intuitive through dynamic video playback, which is easy to arouse learning interest and also reduces the difficulty of psychological preparation for learners.

\subsection{IQiYi}

IQiYi is a high-quality video entertainment website that attracts young users all over the world. The knowledge and education channels of IQiYi are two separate sections, but they contain each other. Although there are two interfaces to enter, knowledge already includes education, and knowledge-related courses can also be found in the education section.

The IQiYi Knowledge Channel was popularized and launched in October 2012. After nearly 9 years of rapid development, IQiYi is in the mature stage of teaching application research [2]. In April 23, 2020, when using "IQiYi video" as the subject, 1142 journal articles were retrieved on CNKI, there is an increase of 10 from the 1132 in 2019; when using "IQiYi video" is the subject, and "education" is the word frequency (including ), 63 journal articles were retrieved. The research, learning and education videos on the "IQiYi video" platform showed a gentle trend.

The content resources of IQiYi Education Channel are very extensive, including practical and test-oriented foreign language learning, children's enlightenment (children's songs and children's English), practical tutorials on sports and fitness, high-quality open classes, academic education, vocational examinations, IT, finance and economics management training and so on. In these subject education and vocational training to meet different ages, the language content of foreign language courses is rich, and the learning is fragmented, so that users can absorb various knowledge points in fragmented time; major vocational examinations and academic courses use IQiYi The professional IT knowledge base in the background allows users to plan their careers in one-stop manner and pass different academic qualification exams; in the financial training curriculum system, they attach importance to combine theory and practice, and continuously improve themselves in practice. The classic channel, IQiYi Education, has launched the training of famous teachers of famous companies. In the practical courses, different fields are involved, and the content of resources is very extensive. What is more, the interest of users of different ages is very important. 


\section{Business Models of Paying for Knowledge of Video Websites: A Case Study from China}

(1) According to the "2019 China Online Video Market Research Report" released by BigData-Research Data Center, IQiYi ranked first in daily active users, of which over 150 million daily active users, followed by Tencent and Youku, and The daily active users of Mango TV fluctuate between 3-4 million.

(2) In China's 2019 online video applications, the total monthly running time of IQiYi users fluctuates between 6 and 7.5 billion hours, ranking first; the total running time of Tencent Video fluctuates between 5 and 6.5 billion hours, Ranked second, while Youku floated between 4-5 billion hours and ranked third.

(3) In 2019, IQiYi's monthly active users of online video APP exceeded 500 million, ranking first; Tencent's second largest number of active users was about 500 million; followed by Youku with slightly less than 500 million active users and ranked third.

The IQiYi video website is a broadcasting platform with high-quality learning video content and a large-scae online learning website. It not only provides simple and easy-tounderstand free learning videos, but also has a paid model, such as VIP membership, which gradually improved Knowledge payment system.

\section{The knowledge-paid business model of the website 3.1. Status quo of knowledge payment model}

In recent years, mobile payment has entered everyone's life. The frequency of users' use has become higher and more widely used. The knowledge payment model for learning products on the website has emerged and developed. Starting from the needs and motivations of consumers, most consumers usually have a very clear willingness to pay, and the payment model they choose is more direct and clear, so most video website platforms provide a variety of knowledge payment business models. It is used to increase the number of users in the long-term and realize the long-term goal of their own platform. Consumers have different learning needs and motivations, and they need different fee models to meet the payment of various knowledge products. Consumers' multi-level and multi-dimensional needs can be met, so the income structure of the video website platform shows diversification and stability [3].

\subsection{Facilitating factors}

The huge number of netizens has laid the foundation for the operation of video websites [3], so the knowledge payment model has become a new profit growth point. The number of about 900 million netizens makes knowledge payment contain huge commercial potential. Most websites attach importance to user experience. Only high-quality video content and differentiated services will take the initiative to pay for learning videos after the learners feel a special experience. Therefore, mainstream video websites have formed a more complete and differentiated membership service system [4]. In the current situation with little difference in content, the use of high-quality content to attract learners to pay for this has become a key factor. The online video industry of China's leading brands provides high-quality video content through capital means to purchase legitimate copyrights, self-created groups continue to launch original videos, create exclusive selfmade content and other core resources, which continue to increase the number of users, video playback and market share Share etc. Based on the huge number of user 


\section{Dongxue Xie}

registrations and usage data, these educational channels use the industry's leading advantages to attract elite educational institutions to the video website platform, allowing users to pay for high-quality and differentiated content.

\subsection{Constraints}

The development time of the paid-for-knowledge business model in China is not short, but people are generally accustomed to the free-to-view model. Most users have relatively low awareness of the protection of domestic genuine video content and willingness to pay for knowledge, and the content homogeneity is serious [5], for example, some paid content on IQiYi videos can be found on Bilibili, so most people do not have a strong desire to pay for this kind of learning video payment, so people's understanding of video content payment is still the initial stage. Therefore, a key factor in attracting users is to improve user satisfaction, while allowing users to more easily access and obtain the content provided by video sites. In order to improve user satisfaction, the transaction cost of the simple website platform and the security of user payment are required [6]. At present, video content is not innovative enough, and even directly imitates and uses excellent foreign programs that consumers like. The market supervision is not in place, and the payment system is not perfect, so that users cannot become buyers or even loyal users of products paid on video websites.

\subsection{Analysis of existing problems}

According to the research data of I-Research on the payment model of short video platforms, among all users surveyed, nearly $30 \%$ of users said that they have never consumed short videos, have no short video payment habits, and $62.27 \%$ of users lack Payment awareness, $32.6 \%$ of users think it is not worth it. Regarding window products, $31.87 \%$ of users believed that the quality of the products did not arouse the desire to buy, while only $7.7 \%$ of users did not find a paid entry. Therefore, short video users did not have the awareness of knowledge payment and needed to enhance their payment habits. The consumption of short video platforms is mainly small-scale, 2-3 times a week. In the past six months, the user's consumption range is between 300-499 yuan, accounting for $35.5 \%$, and the average monthly consumption is 50-80 yuan. The proportion of highconsumption users is small, and there is still room for growth in the consumer market. The frequency of consumption is generally low, only $20 \%$ consume more than four times a week.

Although "education and video" has the potential for profit, video website platforms are trying to seek profit breakthroughs in educational theme content. They still need unique content and continue to strengthen users' habit of paying for courses [7]. Although various surveys have shown that the post-90s' sensitivity to online content has begun to decline, the number of people willing to pay for content has also increased. But at present, most short video users mainly meet their entertainment needs [8]. How to transform video learning into what they just need or how to open the market for those who just need it remains to be further explored.

\section{Conclusion}

This paper introduces the development status of the three major video websites of Bilibili, Tik Tok and IQiYi, as well as the analysis of the learning video status of 


\section{Business Models of Paying for Knowledge of Video Websites: A Case Study from China}

mainstream websites. The website has increased the development of learning videos, high-quality video content and differentiated services of other website platforms, allowing users to actively pay for learning videos. A reasonable price can attract users and increase user viscosity. Analyzed the development status of the learning payment model of mainstream websites and the promotion and restriction factors of this model.

\section{REFERENCES}

1. Y.Li, X.Zheng and Y.Qi, Analysis of the characteristics of the "San Xing Liang Hua" of the "trembling" teaching short video and its enlightenment to the design of microcourses, Journal of Guangxi Vocational and Technical College, 12(06) (2019) 111115.

2. L.Lu, Research on the marketing strategy of iqiyi education channel based on $4 P$ theory, Dissertation of Hebei University, 2017.

3. X.Zhang, Analysis of the Paid Profit Model of Domestic Video Websites: Taking iQiyi as an Example, New Media Research, 5(2) (2019) 10-13.

4. Y.Zhang and S.Zheng, A Comparative Analysis of Marketing Strategies of Beep miles and Tencent Videos, Pay Taxes, 13(33) (2019) 224.

5. L.He, Research on the Promotion Strategy of the Paid Mode of Video Websites, Media Forum, 1(16) (2018) 14-15.

6. Q.Cheng and X.Liu, The development trend and implementation strategy of payment services on video websites, Modern Audiovisual, 3 (2016) 50-53.

7. Y.Wang, Learning is a good "business": A short video platform that has become a learning tool, Modern Audiovisual, 11 (2019) 87.

8. H.Xu and J.Zhang, The Current Situation and Development Trends of China's Online Video Website, Journal of Hengshui University, 4 (2008) 118-120. 